\title{
Spatial orientation: Visual-vestibular-somatic interaction
}

\author{
DONALD E. PARKER, RICK L. POSTON, and WILLIAM L. GULLEDGE \\ Miami University, Oxford, Ohio
}

\begin{abstract}
We are able to orient lines with respect to environmentally or egocentrically defined reference axes. To do this we must be able to compensate for disturbances produced by displacement of our eyes relative to other parts of our bodies and for visual disturbances such as tilted frames or moving visual fields. Compensation signals from somatosensory and vestibular receptors were examined in this investigation. Disturbances were produced by tilting the head and by rotating a large visual display. Compensation signals associated with gravity were manipulated by placing the observers horizontally on a board or seating them vertically. Experiment 1 examined effects of visual disturbance on the ability of supine observers to set a line to the longitudinal body axis while the head was tilted toward one shoulder or while the head was straight. Effects of the visual disturbance were greater when the head was tilted than when it was straight. This indicates that the effects of visual disturbance were greater for a task that required compensation. Experiment 2 compared the performance of supine and erect observers. No differences between the performance of observers on a task requiring the use of compensation signals were obtained under these two conditions. This suggests that enrichment of compensatory signals did not reduce the effects of visual disturbance. Experiment 2 did replicate the result from Experiment 1 that the effects of visual disturbance were greater when observers performed a task that required them to use compensation signals. Finally, Templeton's (1973) previous report that supine observers undercompensate for head tilt was replicated.
\end{abstract}

Perception of orientation in space and of selfmotion depends on information provided by visual, vestibular, and somatosensory receptors. Given the validity of this premise, a basic question is apparent. How is information from the different receptors integrated? Among the ancillary questions that derive from the basic one are the following. Does the relative importance, or "weighting," of the information from the different receptors change during normal activity? How is the integration process modified when information from the different receptors is in conflict? The research described in this paper addresses these questions.

An erect observer in a darkened environment is able to set a light line parallel to gravity. Errors in the settings are introduced when the observer's head or entire body is tilted in the frontal plane. Head or body tilt can be described as a disturbance of the visual signal from the light line. Following body tilt, a vertically oriented line no longer produces an image on the retina's longitudinal axis. In order to set the line to gravity, the observer must compensate for the disturbance. Information indicating the direction of

This work was supported by Contract 9-14538 between Miami University and the National Aeronautics and Space Administration. We thank Susan Pope, Jennifer Mattox, Robert Shorey, and Gabriell Michel for their assistance. Our mailing address is: Department of Psychology, Miami University, Oxford, Ohio 45056. gravity that can be used to compensate for the disturbance is transduced by vestibular and somatosensory receptors. Analysis of errors in line settings following a disturbance allows investigators to characterize a sensory integration process.

Numerous investigators have examined the effects of various disturbances and/or manipulations of compensation signals on spatial orientation perception. Two main types of disturbances have been studied. First, observers' heads or bodies have been tilted or rotated and they have been required to align a target line with an axis different from the head axis. Second, visual disturbances have been produced by tilted frames, striped backgrounds, and large, rotating visual displays. Compensation signals have been manipulated and studied by changing the orientation of the observers with respect to gravity, particularly by comparing the performance of erect and supine observers. It should be noted that postural tilt can be viewed both as a disturbance and as a technique for manipulating compensation signals. The magnitude of the tilt determines the magnitude of the disturbance that the observer attempts to compensate. The strength of the gravity information available to the observer, particularly that derived from the vestibular otolith receptors, also varies with tilt (Lechner-Steinleitner \& Schöne, 1980).

Many studies have examined disturbance of the perceived vertical associated with tilting an observer's head or body to an off-vertical axis. Al- 
though there is disagreement with regard to specific values and conditions, small angles of body tilt are associated with overcompensation (i.e., the subjective vertical is displaced in the direction opposite to the body tilt), and large angles of body tilt are associated with undercompensation (Day \& Wade, 1969; Howard \& Templeton, 1966).

Disturbance of the perceived vertical associated with additional visual cues also has been examined extensively. If a vertical line is surrounded by a large, laterally tilted frame, the observer may report that the line is tilted in the direction opposite to that of the frame (Witkin, Dyk, Faterson, Goodenough, \& Karp, 1962/1974). Similar effects have been obtained when the target line is placed over a striped background (Bischof \& Scheerer, 1970; LechnerSteinleitner \& Schöne, 1980). The apparent orientation of a line can be disturbed also by a large visual display rotating around the observer's line of sight. The rotating display induces tilt of the target line in the direction opposite to display rotation (Dichgans, Held, Young, \& Brandt, 1972).

Compensation signals have been manipulated employing a procedure developed by Rock (1954). The orientation performance of erect and supine observers has been compared. Erect observers set a target line to the gravitationally defined vertical, whereas supine observers set the target line to a bodydefined axis. When the target line was located in the supine observer's frontal plane, gravity cues transduced by vestibular and somatosensory receptors could not be used by the observers to accomplish the task.

Effects of combinations of disturbances have been evaluated by examining the ability of observers to set a target line to a specified axis while the observers were tilted and were being exposed to additional visual input. Witkin and Asch (1948) reported that the effect of a tilted frame was greater when the body was also tilted off-vertical. Bischof and Scheerer (1970) reported that the effects on the perceived vertical of a very slowly rotating striped visual field varied with body tilt. Maximum disturbances from the striped field were associated with body tilts between 60 and $90 \mathrm{deg}$ off-vertical. Dichgans, Diener, and Brandt (1974) reported that the perception of tilt induced by a large, moving visual display increased as a function of head tilt. Young, Oman, and Dichgans (1975) indicated that the effects on perception of self-motion associated with a moving display in the peripheral visual field were larger when observers were rolled to a 90-deg orientation or were inverted than when they were upright.

Manipulation of compensation signals by placing observers in the supine position has been combined with visual disturbance in a few investigations. The effects of a tilted frame were greater when an overhead display in the horizontal plane was viewed by a supine observer than when a vertical display was viewed by an erect observer (Brosgole \& Cristal, 1967; Goodenough, Oltman, Sigman, \& Cox, 1981). On the other hand, Finke and Held (1978) reported smaller effects of a large, rotating visual display when observers were supine than when they were upright.

Apparently, only Templeton (1973) has examined previously the combination of visual and head-tilt disturbances with observers in erect and supine positions. His study confirmed previous observations that the effects of a visual disturbance from a tilted frame were greater for supine than for erect observers. For supine observers, the effects of the tilted frame were the same or less when the observers' heads were tilted than when their heads were straight. This finding differs from what might have been expected based on previous reports employing erect observers. It is consistent, however, with the quite small effect of a tilted frame on erect observers whose heads were tilted in Templeton's study. Finally, Templeton's report indicates that supine observers whose heads were tilted undercompensated for the head tilt when they attempted to set a line to their longitudinal body axes in the absence of a disturbance from the frame.

\section{EXPERIMENT 1}

Previous research had indicated that the ability of observers to set a target line to a specified axis was disturbed by a large, rotating visual field. The primary question addressed in this experiment is "Are the effects of the moving display greater when the observer performs a task that requires the use of compensation signals than when no compensation is needed to perform the task?" For the supine observer whose head is straight, retinal signals alone should provide the information needed to set a target line to the longitudinal (z) body axis. Compensation signals from neck somatosensory receptors indicating the angle of head tilt relative to the body trunk should be required for the supine observer whose head is tilted to perform this task. The question becomes "Are the effects of the moving visual display greater when the supine observer sets the line to the $\mathrm{z}$ body axis with the head tilted than with the head straight?

\footnotetext{
Method

Observers. Six students, three men and three women, completed Experiment 1 for payment. All were familiar with the tasks of setting a line to the $z$ head axis and the $z$ body axis as a consequence of participation in previous experiments. They were not familiar with the moving visual display or with the literature concerning the effects of moving peripheral visual fields on perceptual orientation.

Appantus. For all trials, the observers was supine on the board. The observer's shoulders were restrained by shoulder pads, and the head rested in an oval padded cloth ring. This ring served to main-
} 
tain the observer's head position and raised the head approximately $2.5 \mathrm{~cm}$ above the surface of the board. The ring was positioned so that the head was either straight or tilted 30 deg toward the left shoulder. Two 2-cm "half-round" wooden strips $35 \mathrm{~cm}$ long and $5 \mathrm{~cm}$ apart were attached to the board so that they started at the level of the shoulder pads and ran down the board along each side of the spine. These strips served to align and maintain the trunk at a 90-deg angle to the shoulders. The feet touched opposite sides of a $2-\mathrm{cm}$ board mounted on edge in alignment with the $z$ body axis.

The target stimulus was a $24 \times .5 \mathrm{~cm}$ luminous line painted on a $35-\mathrm{cm}$ diameter black cardboard disk. The disk was attached to a servomotor and mounted so that the display was in a plane parallel to the floor and at a viewing distance of $60 \mathrm{~cm}$. For all trials, the target line's axis of rotation was directly over the observer's nose. The observer controlled the rotation of the stimulus line by a knob on a control servomotor. The investigator read the setting of the target line from a dial on a third servomotor.

The large, moving visual display was provided by a cone. Luminous paint stripes of about 7-deg width covered the inside wall of the cone. The base of the cone was $61 \mathrm{~cm}$ in diameter, and the truncated apex was $43 \mathrm{~cm}$. The retinal projection of the lower edge of the cone subtended about $150 \mathrm{deg}$. The base of the cone was suspended $8 \mathrm{~cm}$ above the observer's head. The cone could be rotated around its axis with a dc motor through a pulley and belt. The disk that carried the luminous target line was suspended from the cone apex. Relative to the observer, the cone could be rotated clockwise or counterclockwise.

Procedure. Observations were performed with the observer lying horizontally (supine). Each observer completed four experimental sessions consisting of 24 trials each. For 12 trials the head was tilted 30 deg toward the left shoulder, and for the remaining 12 trials the head was straight. For one-half of the trials the observer set the light line to the $z$ body axis, and for the remaining one-half he or she set the line to the $\mathrm{z}$ head axis. The reference axis for a particular trial was indicated to the observer by tones delivered through a headset. Three cone-rotation conditions were used: clockwise, counterclockwise, and stationary. Each set of conditions was repeated once during a session. A typical session was as follows: with the head left and the cone rotating clockwise, the observer set the light line twice to the $\mathrm{z}$ body axis and twice to the $z$ head axis (alternating); this was followed by four settings during each of the remaining two cone-rotation conditions. Then the head tilt was changed and the sequence of the first 12 trials was repeated. The order of initial head tilts, rotations, and referencesetting commands across sessions was partially counterbalanced and randomized. The target was moved directly above the observer's nose in each head position. Cone-rotation rate was $60 \mathrm{deg} / \mathrm{sec}$. This rotation rate was chosen during preliminary observations as that which would produce maximum subjective effects and was just below blur threshold. It corresponded to the rate used in previous observations (Dichgans \& Brandt, 1978; Dichgans, 1972).

The luminous paint was excited prior to each session and faded throughout the session. Because the observer dark-adapted during the trials, the apparent brightness of the line and the stripes did not change greatly.

\section{Results}

Mean luminous line settings for the two head positions (straight or tilted left) and the two reference axes ( $\mathrm{z}$ body and $\mathrm{z}$ head) as a function of the three cone-rotation conditions are illustrated in Figure 1. A luminous line setting of $360 \mathrm{deg}$ corresponded to the observer's $z$ body axis (and to the $z$ head axis when the head was straight).

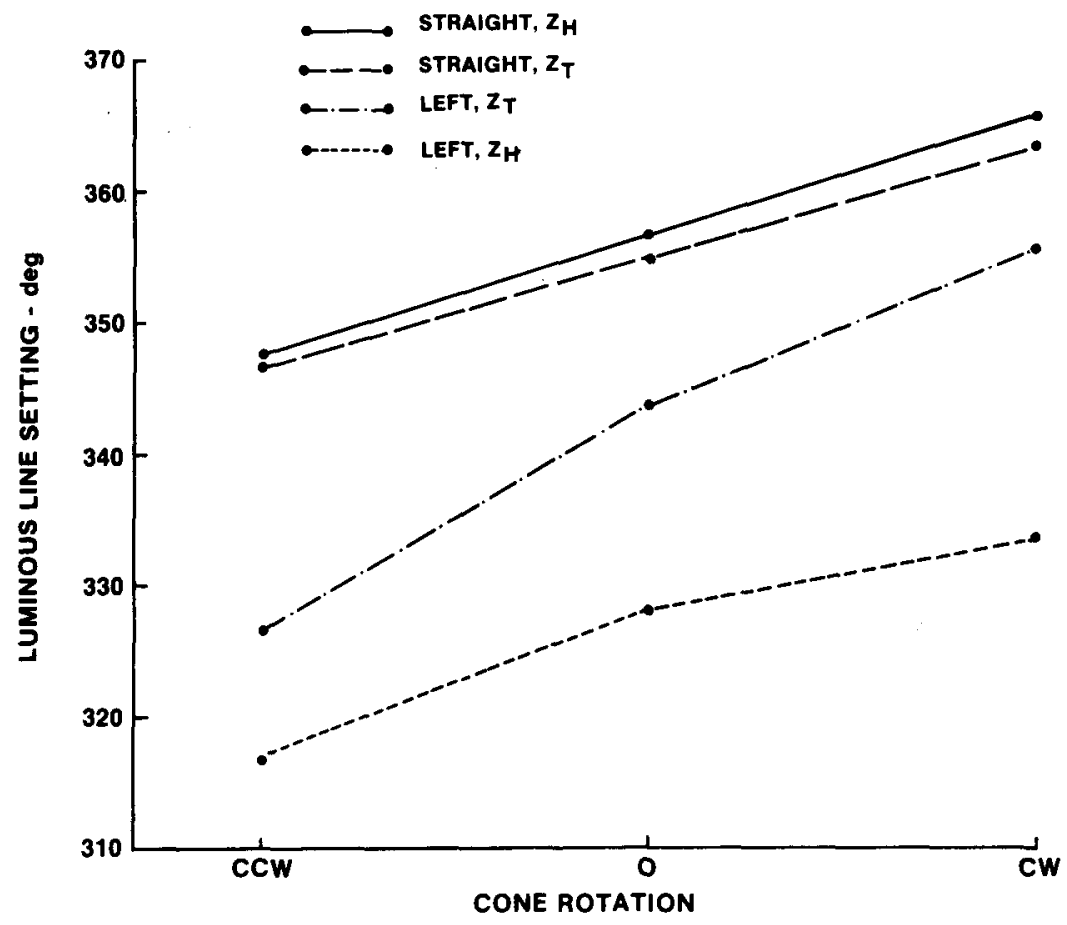

Figure 1. Mean luminous line settings as a function of cone rotation. Separate curves are plotted for the four combinations of head tilt (straight and left) and reference axis ( $z$ head $-z_{H} ; z$ body $-z_{T}$ ). A luminous line setting of 360 deg corresponded to the observer's longitudinal body axis. The cone was stationary $(0)$, rotated counterclockwise $(C C W)$, or rotated clockwise $(\mathrm{CW})$ relative to the observer. The observens were placed in the supine body position. 
The primary finding from Experiment 1 was that the effects of cone rotation were greater when observers whose heads were tilted set the line to their $z$ body axes than when they performed the same task with the head straight. With the head tilted, the difference between $\mathrm{z}$ body axis settings during clockwise and counterclockwise cone rotations was $27.7 \mathrm{deg}$. When the head was straight, this difference was only $16.4 \mathrm{deg}$. The difference of $11.3 \mathrm{deg}$ in the effect of cone rotation was statistically significant $[t(23)=4.3$, $\mathrm{p}<.001]$.

Line settings to the $z$ body axis deviated in the direction of head tilt. With the cone stationary, mean $z$ body axis settings were $343.4 \mathrm{deg}$ when the head was tilted to the left and $355 \mathrm{deg}$ when the head was straight. The difference between these means appears to have been reliable also $[\mathrm{t}(3)=12.4, \mathrm{p}<.002]$.

The data were analyzed also with a three-way (head tilt $\times$ reference axis $\times$ cone rotation) analysis of variance. The statistically significant head position $\times$ reference axis interaction $[F(1,5)=17.79, p<$ $.008]$ indicates that the observers were able to perform the basic task. Cone rotation strongly influenced line settings $[F(2,10)=36.87, p<.0001]$. As illustrated in Figure 1, the settings deviated in the direction of the rotation. Examination of the data indicates that the effects of cone rotation were greater when the head was tilted toward the left shoulder than when the head was straight $[F(2,10)=7.53$, $p<.015]$. Also, the effects of cone rotation were greater when the reference axis was the $\mathrm{z}$ body axis than when it was the $z$ head axis $[F(2,10)=6.6$, $\mathrm{p}<.02]$.

\section{Discussion}

The finding that cone-rotation effects were larger with the head tilted than when it was straight when observers set the luminous line to the $\mathrm{z}$ body axis indicates that visual disturbance more strongly affected a task that required compensation than it affected one in which information from a single receptor was sufficient to accomplish the task. This observation is consistent with the numerous reports noted in the introduction that the effects of visual disturbance were greater when the observers were tilted to an offvertical axis than when they were erect. Templeton's (1973) report indicating no differences in the effects of a visual distrubance across head-tilt conditions with supine observers appears to be at variance with the previous findings on off-vertical tilt effects as well as with the results of the present study.

The results of Experiment 1 indicate also that the observers undercompensated for head tilt when they attempted to set the line to the $z$ body axis. This finding replicates that reported by Templeton (1973). The magnitude of the undercompensation $-16.6 \mathrm{deg}$ relative to the board-defined $z$ axis or $11.6 \mathrm{deg}$ relative to $z$ body axis settings with the head straightwas greater than that reported by Templeton or that obtained by us in preliminary studies. The large effects found in Experiment 2 may reflect differences across observer populations in the weighting of spatial-orientation signals.

The effects of the moving visual display were similar in both direction and magnitude to those reported previously by Dichgans et al. (1972) for upright observers. Cone rotation elicited an interesting pattern of subjective experience. Initially, the striped walls of the cone appeared to rotate and the luminous line on the disk remained stationary. After a very brief period (1-10 sec), the luminous line appeared to rotate in the direction opposite to the cone rotation. This was equivalent to displacing the reference axis in the direction of rotation. Although the luminous line did not appear to change orientation with respect to the observer, the sensation of self-motion was not strong.

Without measurements of neck length or photographs of the observers in the apparatus, the exact position of the head during head tilt cannot be determined. Consequently, it is not meaningful to consider errors in luminous line settings when the observers' heads were tilted and the observers set the line to the $\mathrm{z}$ head axis. Nevertheless, responses when the $\mathrm{z}$ head axis was the reference axis may be of interest for qualitative comparison and are presented in Figure 1. Moreover, the requirement that observers alternated in their line settings between the $z$ head and $z$ body axes may have served as a distraction and kept them from perseverating in a response that differed from their actual perception.

\section{EXPERIMENT 2}

Experiment 1 demonstrated that the effects of a disturbing visual input were greater when the orientation task required observers to integrate sensory information from retinal and neck somatosensory receptors. In comparison with the supine observer, an erect observer whose head is tilted and who is required to set $\mathrm{a}$ target line parallel to the $\mathrm{z}$ body axis has additional compensatory information. In this second case, information concerning the direction of gravity transduced by vestibular and somatosensory receptors can be combined with somatosensory information indicating the angle of head tilt relative to the torso. The major question addressed in Experiment 2 is "Does additional compensatory information reduce the effects of visual disturbance?" In terms of the specific procedures employed, this question becomes "Are the effects of cone rotation the same when erect observers whose heads are tilted set the luminous line to their $\mathbf{z b o d y}$ axes as when supine observers whose heads are tilted perform the same task?"

\section{Method}

Observers. Six students, three women and three men, who were 
familiar with the task and procedures as a result of having participated in previous studies, completed Experiment 2 for payment.

Appiratus. The apparatus used when the observers were horizontal was the same as that for Experiment 1. For trials with the observers vertically oriented, the cone and target disk were rotated $90 \mathrm{deg}$ so that the longitudinal cone axis was parallel to the floor. The observers sat on an adjustable stool, and their heads were held in a headrest with elastic bands. Rigid bars were placed between the observer's torso and arms to help maintain an upright posture.

Procedure. Each observer completed four experimental sessions. A session consisted of 32 trials, 16 in the supine orientation and 16 while the observer was erect. The observers set the luminous line to their $\mathbf{z}$ head or $\mathbf{z}$ body axes with their heads either straight or tilted toward their left shoulders. Two cone-rotation conditions were used: stationary or rotating clockwise at $60 \mathrm{deg} / \mathrm{sec}$. For a particular set of conditions, each observation was repeated once. All of the trials for a particular postural orientation were completed before proceeding with the second orientation. Order of trials across sessions was partially counterbalanced and randomized. In contrast with Experiment 1, the cone and target were centered with respect to the observer's nose for the head-straight condition; target position was not changed for the head-left condition.

\section{Results}

Mean luminous line settings calculated across observers and sessions as a function of head tilt are illustrated in Figure 2. The eight curves are associated with the manipulations of body position, reference axis, and cone rotation. A luninous line setting of 360 deg corresponded to the observer's $z$ body axis when supine or the $\mathbf{z}$ body axis and gravity when erect.

No differences in cone rotation effects were found across the conditions related to the primary question addressed in Experiment 2. Cone-rotation effects (the differences between the rotation and no-rotation conditions) were virtually the same when supine observers whose heads were tilted set the line to their $z$ body axes (11.7 deg) as when erect observers whose heads were tilted performed the same task $(11.6 \mathrm{deg})$.

The effects of cone rotation were greater when the observers' heads were tilted and they set the line to their $\mathrm{z}$ body axes than when their heads were straight. For the case in which the observers were supine, rotation resulted in an 11.7-deg shift in the settings when the heads were tilted and an 8.3-deg shift when the heads were straight. This 3.4-deg difference appears to be reliable $[t(23)=2.83, p<.01]$. The greater effect of cone rotation when the head was tilted than when it was straight was obtained also when the observers were erect. When erect observers set the line to their $z$ body axes, rotation resulted in an 11.6-deg shift with the head tilted and only a 5.5-deg shift with the head straight. This 6.1-deg difference appears to be reliable also $[\mathrm{t}(23)=5.61, \mathrm{p}<.001]$.

One additional cone-rotation effect was obtained. Under the head-straight condition, supine observers exhibited a greater effect of cone rotation (8.3 deg) than did erect observers $(5.5 \mathrm{deg})$. This 2.8 -deg difference associated with cone rotation also appears to be reliable $[t(23)=2.84, p<.01]$.

Under the no-rotation conditions, luminous line settings to the body axis with the head tilted toward

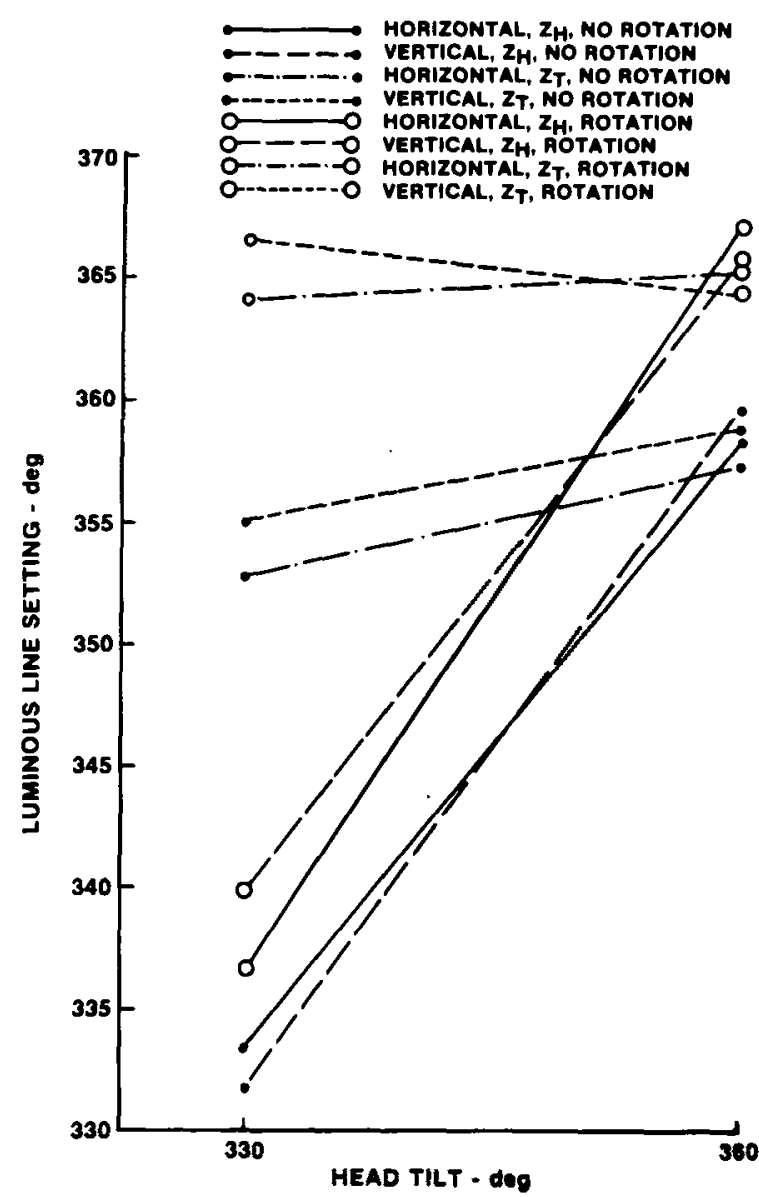

Figure 2. Mean luminous line settinga w a function of bead tilt. Separate curves are plotted for the elght comblnations of body position (supine-horizontal; erect-vertical), reference axis (z head $-z_{1} ; z$ body $\rightarrow z_{T}$ ), and cone rotation (no rotation and rotation clockwise). The observer's head was atraight (360 deg) or tilted 30 deg toward the left shoulder ( 330 ded). Luminous line settings of 360 deg corresponded to the longitudinal body axis as well as to gravity when the observer was vertically oriented.

the left shoulder averaged $354.7 \mathrm{deg}$ when the observers were supine and $357 \mathrm{deg}$ when they were seated vertically. The difference of $2.3 \mathrm{deg}$ in settings was statistically significant $[\mathrm{t}(23)=2.51, \mathrm{p}<.02]$.

The data obtained in Experiment 2 also were analyzed with a four-way (head tilt $x$ reference axis $x$ cone rotation $x$ body position) analysis of variance. Cone rotation produced deviation in the luminous line settings in the direction of the rotation $[F(1,5)=$ 45.33, $\mathrm{p}<.001]$. The large effects associated with head tilt $[F(1,5)=118.34, p<.0001]$ and reference axis $[F(1,5)=50.64, p<.001]$, as well as with the head tilt $\times$ reference axis interaction $[F(1,5)=30.54$, $\mathrm{p}<.003$ ], indicate that the observers were able to perform the basic task.

\section{Discussion}

Essentially the same effects of the visual disturbance were found across the supine and erect con- 
ditions when observers whose heads were tilted performed a task that required the use of nonretinal compensation signals. The addition of potential compensatory signals associated with the gravity stimulus transduced by the somatosensory and vestibular otolith receptors apparently did not reduce the effects of cone rotation. This finding is puzzling because of the difference in the effects of cone rotation between supine and erect observers whose heads were straight. In this latter case, gravity signals did appear to reduce the effects of cone rotation.

The results of Experiment 2 confirmed the major finding from Experiment 1 . The effects of cone rotation were greater when supine observers set the line to their $z$ body axes with their heads tilted than when their heads were straight. This again supports the view that the effects of the disturbance produced by the rotating visual field were greater when the task required the observer to integrate a compensation signal from neck somatosensory receptors with the retinal signal than when the retinal signal alone provided information sufficient to accomplish the task.

The results of Experiment 2 support the observation from Templeton (1973) that addition of gravity signals by placing the observer in the erect position improves compensation for head tilt. In Experiment 2 , the change in compensation associated with the addition of gravity information, although statistically significant, was only about $2 \mathrm{deg}$, whereas the effects reported by Templeton and seen by us in preliminary experiments were approximately 6 deg. The difference between previous results and those obtained in Experiment 2 can be attributed primarily to one observer who exhibited greater undercompensation in the erect position that in the supine position. Caution in interpreting this observation is indicated because the retinal projection of the target differed between the head-tilted and the headstraight conditions. In contrast with Experiment 1, the target position was not changed for the headtilted and head-straight conditions.

\section{GENERAL DISCUSSION}

\section{Models}

The concepts of disturbance and compensation described in the introduction derive from observations on animals such as cephalopods and rodents. In these animals, disturbances such as body tilt with respect to gravity may elicit reflex movement of the eyes, including ocular countertorsion (counterrolling). These reflexes serve to stabilize the eye so that the location on the retina of an image from an environmental stimulus is invariant with head tilt. Bischof (1974) has labeled the relatively complete compensation achieved by reflex movement of the receptor "external compensation." Insofar as countertorsion is concerned, external compensation pro- cesses oppose the effects of head-tilt disturbances to only a minor degree in human beings. Numerous reports indicate that the maximum ocular countertorsion is only about 6-8 deg (see Diamond, Markham, Simpson, \& Curthoys, 1979). Moreover, the results of a study by Wolfe and Held (1979) suggest a separation between the neural mechanisms underlying perceived tilt and those associated with ocular countertorsion. To the extent that the performance of human beings cannot be attributed to reflex stabilization of a receptor, an "internal," neural compensation process must be postulated (Bischof, 1974).

Several investigators have proposed information flow models to account for an internal compensation process that requires sensory integration. Gibson (1966) introduced the concept of a spatial orientation perceptual system and suggested that this system receives inputs from visual, vestibular, and somatosensory receptors. Templeton (1973) diagramed the information links among a light bar stimulus, the eyehead system, the trunk, a visual frame, and gravity. Ebenholtz (1977) proposed a flow chart to represent points of information integration in an "algorithmprocessing model" of perceptual orientation. Finally, Bischof (1974) and Mittelsteadt (1964) have proposed "mesh" ("feed-forward") models to account for the compensation processes underlying perceptual orientation behavior and have attempted to quantify parts of their models.

A simple approach to modeling sensory integration would be to suggest that effects associated with disturbances are additive. For example, effects associated with disturbances such as head tilt or a rotating visual display could be determined separately. A simple additive model would suggest that disturbance due to head tilt would sum with disturbance due to display rotation.

Previous research has shown a simple additive model to be inadequate. As noted in the introduction, effects of tilted frames, striped backgrounds, and large rotating displays are greater when observers are in an off-vertical postural orientation than when they are erect.

The consistent reports that the effects of visual disturbance varied as a function of body position suggest that these effects depend on the quality and magnitude of compensation signals from vestibular and somatosensory receptors. For the erect observer, both somatosensory and vestibular receptors transduce gravity information; this information is not available to supine observers. As suggested by von Holst (1950), perhaps the "weighting" given to gravity information and visual information changes under a variety of conditions, including alterations of body position.

Comprehension of the process underlying the weighting changes for different sensory inputs under different conditions may be facilitated by considera- 
tion of another type of disturbance, head tilt relative to trunk and an additional class of compensation information, somatosensory receptor signals indicating head tilt relative to the trunk. Templeton (1973) has examined this general situation and noted that for supine observers required to set a line to their $z$ body axes, the effects of a frame tilted in the observer's frontal plane were the same or less when the head was tilted relative to when the head was straight. The implication of Templeton's finding is that the visual disturbance produced by the frame was independent of the requirement for a compensation signal to accomplish the task. The thrust of the previous studies seems to run counter to Templeton's observations.

The major finding of the present paper also is at variance with Templeton's findings. For supine observers required to set the line to their $z$ body axes, a greater effect of cone rotation was seen when the head was tilted than when the head was straight. This seems to suggest, in contrast with Templeton's findings but congruent with other findings, that the effects of a visual disturbance are greater when compensatory information is required for an observer to perform an orientation task.

Also in contrast with Templeton's findings, but not easily interpretable in view of compensatory information available, was the finding in the present investigation that the disturbance associated with the rotating cone was essentially the same across the erect and supine conditions when the observers' heads were tilted and they set the line to their $\mathrm{z}$ body axes. This is particularly puzzling in view of Templeton's finding, confirmed by the present investigation, that observers undercompensated for head tilt in the supine but not in the erect postural-orientation condition.

The view that the quality and magnitude of compensatory information available determines the effect of a disturbance is consistent with the observation that cone rotation produced a greater effect when the observers weie supine and their heads were straight than when they were erect and their heads were straight. While no compensatory information is required to perform the task, at least redundant information indicating the direction of the $z$ body axis is available in the erect condition from gravity transducers, whereas this is not true in the supine case.

In conclusion, advancement of sensory integration models may depend on future conceptual developments and empirical research to resolve the discrepancies among the findings from the present investigation as well as between the results of this investigation and those reported by others. Recent research on spatial orientation does suggest, however, that a continued effort to formulate research questions and interpret findings within the framework of disturbance/compensation mechanisms may facilitate development of more adequate sensory integration models.

\section{Limitations}

Differences between the results from the present investigation and those reported previously may be attributed to a variety of factors, including changes in receptor activity over time and differences between the stimuli used to produce visual disturbance. Several investigators have demonstrated changes in orientation performance over time (see Day \& Wade, 1966, and Schöne \& Lechner-Steinleitner, 1978). Temporal variation in performance was not evaluated in the present study.

The static frame used by Templeton and others is likely to evoke activity in different receptors from a wide-field moving display. Observation on the responses of vestibular nucleus neurons, such as that by Waespe and Henn (1979) indicating that the same neuron responds to angular acceleration affecting the semicircular canal and a rotating visual field in a completely appropriate manner, suggests that the best opportunity to understand sensory integration and compensation for visual disturbance is provided by studying the effects of large, moving visual displays that affect the peripheral visual field.

The data illustrated in Figures 1 and 2 are not representative of all of the observers who participated in these experiments. In one preliminary study of neck-vibration effects, students were identified who exhibited statistically significant overcompensation when aligning the luminous line with their $z$ body axes while supine with their heads tilted toward their shoulders. Other observers showed very little under- or overcompensation under these conditions.

All of the observers who participated in the experiments described in this paper were familiar with the task of orienting a luminous line to different body axes as a consequence of their experience in previous studies. While the responses did vary across sessions (days), statistically significant main or interaction effects associated with sessions were not seen in preliminary studies.

Feedback concerning the "correct" setting for the luminous line was not provided. The results from a recently completed study employing a different group of observers indicated that statistically significant changes in orientation performance can occur very rapidly (within the first session) if feedback is provided.

Part of the variability across individuals seen in the present experiments may be attributable to the manner in which the observers' heads were moved. In these experiments, the investigator changed the position of the head restraint (ring or head holder) and the observers actively moved their heads to the altered position. The results of a recently completed 
experiment indicate that the responses were less variable when the observers' heads were passively moved very slowly from one position to another.

\section{Space Motion Slckness}

The research described in this paper is part of an effort to address the problem of space motion sickness. About $50 \%$ of Apollo, Skylab, and Space Shuttle astronauts have experienced motion sickness symptoms during weightlessness. Although not lethal, space motion sickness is expensive. Costs associated with loss of up to $25 \%$ of an astronaut's working time due to motion sickness during a shuttle mission have been estimated in the millions of dollars.

The most widely shared view concerning space motion sickness etiology is the sensory conflict (mismatch) theory (Reason \& Brand, 1975). This theory suggests that conflict between the information provided by the various spatial orientation receptors leads to motion sickness. A predictive sensory conflict theory of space motion sickness requires understanding of how sensory information is integrated, of how individuals differ, and of how the integration processes can be modified. Observations such as those described in this paper may allow prediction of space motion sickness susceptibility and development of training procedures to help alleviate this problem.

\section{Conclusions}

The major conclusions that derive from this investigation are as follows:

(1) The effects of a visual disturbance were greater when observers performed a task that required multisensory compensation than when no compensation was required.

(2) No differences were noted in the effects of visual disturbance on performance requiring compensation when compensatory signals were enriched by placing observers in the vertical body position relative to the situation in which compensatory information was limited because observers were placed in the supine position.

(3) Templeton's (1973) report that supine observers undercompensate for head tilt was replicated.

\section{REFERENCES}

Brschor, N. Optic-vestibular orientation to the vertical. In $\mathbf{H} . \mathbf{H}$. Kornhuber (Ed.), Handbook of sensory physiology (Vol.6/2). Berlin: Springer, 1974.

Bischor, N., \& Scheeren, E. Systemanalyse der optischvestibulären Interaktion bei der Wahrnehmung der Vertikalen. Psychologisch Forschung, 1970, 34, 99-181.

Brosgole, L., \& Cristal, $R$. M. The role of phenomenal displacement on the perception of the visual upright. Perception \& Psychophysics, 1967, 2, 179-188.

DAY, R. H., \& WADE, N. J. Visual spatial after-effect from prolonged head tilt. Science, 1966, 1S4, 1201-1202.
DAY, R. H., \& WADE, N. J. Mechanisms involved in visual orientation constancy. Psychological Bulletin, 1969, 71, 33-42.

Diamond, S. G., Markhay, C. H., Simpson, N. E., \& Curthoys, I. S. Binocular counterrolling in humans during dynamic rotation. Acta Otolaryngologica, 1979, 87, 490-498.

Dichoan8, J., \& Brandt, T. Visual-vestibular interaction: Effects on self-motion perception and postural control. In R. Held, H. Leibowitz, \& H. L. Teuber (Eds.), Handbook of sensory physiology (Vol. 8). Berlin: Springer-Verlag, 1978.

Dichonns, J., Diener, H. C., \& Brandt, T. Optokineticgraviceptive interaction in different head positions. Acto Otolaryngologica, 1974, 78, 391-398.

Dichanes, J., Held, R., Youna, L., \& Brandt, T. Moving visual scenes influence the apparent direction of gravity. Science, 1972, 178, 1217-1219.

Ebenholtz, S. M. The constancies in object orientation: An Algorithm processing approach. In W. Epstein (Ed.), Stability and constancy in visual perception. New York: Wiley, 1977.

Finke, R. A., \& Held, R. State reversals of optically induced tilt and torsional eye movements. Perception \& Psychophysics. $1978,23,337-340$.

Gibson, J. J. The senses considered as perceptual systems. Boston: Houghton Mifflin, 1966.

Goodenovgh, D. R., Olmuan, P. K., Sigman, E., \& Cox, P. W. The rod-and-frame illusion in erect and supine observers. Perception \& Psychophysics, 1981, 29, 365-370.

How ARD, I. P., \& TEMPLETON, W. B. Human spatial orientation. London: Wiley, 1966.

Lechne R-Ste inleitiner, S., \& Schöne, H. The subjective vertical under "wet" and "dry" conditions at clockwise and counterclockwise changed positions and the effect of parallellined background field. Psychological Research, 1980, 41, 305-317.

Mitrelstendt, H. Basic solutions to the problem of angular orientation. In R. F. Reiss (Ed.), Neural theory and modeling. Palo Alto: Stanford University Press, 1964.

Reason, J. R., \& Brand, J. J. Motion sickness. London: Academic Press, 1975.

Rock, I. The perception of the egocentric orientation of a line. Journal of Experimental Psychology, 1954, 48, 367-374.

Schöne, H., \& Lechner-Steinleitwer, S. The effect of preceding tilt on perceived vertical. Acto Otolaryngologica, 1978, 85, 68-73.

Templeton, W. B. The role of gravitational cues in the judgment of visual orientation. Perception \& Psychophysics, 1973, 14, $451-457$.

von Hozst, E. Die Arbeitsweise des Statolithenapparates bei Fischen. Zeitschrift für vergleichen Physiologie, 1950, 32, 60-120.

WAEspe, W., \& Hens, V. The velocity response of vestibular nucleus neurons during vestibular, visual, and combined angular acceleration. Experimental Brain Research, 1979, 37, 337-347.

Witrin, H. A., \& Asch, S. L. Studies in space orientation: IV. Further experiments on perception of the upright with displaced visual fields. Journal of Experimental Psychology, 1948, 38, 762-782.

Witkin, H. A., Dyx, R. B., Faterson, H. G., Goodenough, D. R., \& KARP, S. A. Psychological differentiation. Potomac, Md: Erlbaum, 1974. (Originally published by Wiley, 1962).

Wourz, J. M., \& HewD, R. Eye torsion and visual tilt are mediated by different binocular processes. Vision Research, 1979, 19, 917-920.

Youno, L. R., Oman, C. M., \& Dichanes, J. M. Influence of head orientation on visually induced pitch and roll sensation. Aviation, Space and Environmental Medicine, 1975, 46, 264-268.

(Manuscript received November 9, 1981; revision accepted for publication November 9,1982 .) 